\title{
A Simple and Mild Microwave-Based Synthesis of Novel Function- alized Benzofulvenes
}

\author{
Adam C. Glass*a \\ Katherine E. Caspary ${ }^{b}$ \\ Cole Fisher ${ }^{\mathrm{a}}$ \\ Connor Whyte ${ }^{a}$ \\ James Okubo ${ }^{\mathrm{a}}$ \\ Lev N. Zakharovc \\ a Department of Chemistry, Pacific Lutheran University, 1010 \\ 122st S, Tacoma, WA 98447, USA \\ glassac@plu.edu \\ b Department of Chemistry, Oregon State University, 153 Gil- \\ bert Hall, Corvallis, OR 97331, USA \\ ' CAMCOR, University of Oregon, 1443 E. 13th Avenue Eugene, \\ OR 97403, USA
}

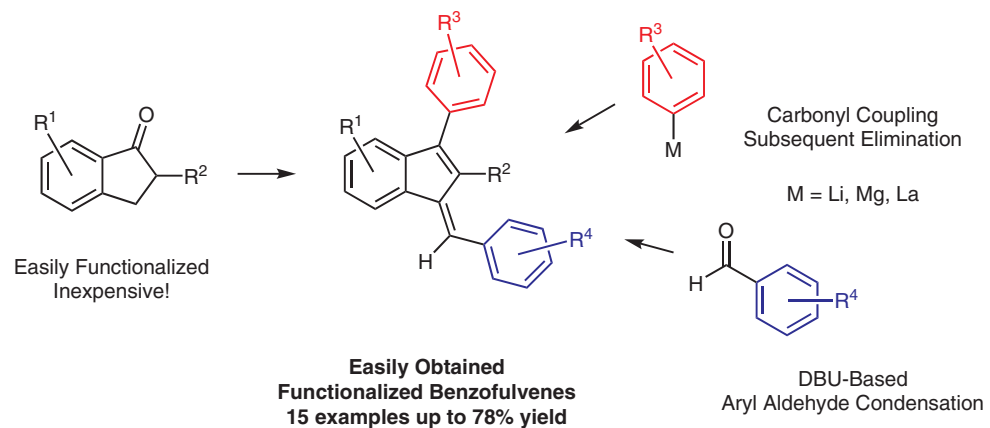

Received: 01.08.2018

Accepted after revision: 21.08.2018

Published online: 14.09 .2018

DOI: 10.1055/s-0037-1610366; Art ID: so-2018-d0045-psp

License terms: (C)

Abstract Benzofulvenes and their derivatives are useful molecular entities having applications as biologically active molecules, polymer precursors, and optoelectronic devices. We have developed a simple and mild synthetic method for the formulation of a variety of these interesting compounds. Using carbonyl coupling techniques combined with microwave heating, a wide variety of functionalized benzofulvenes can be accessed rapidly in good yield. Furthermore, we have obtained five crystal structures further expanding a limited number of benzofulvene structures available.

Key words aromaticity, benzofulvenes, DBU, microwave, mild

Fulvene-based motifs are widely regarded as molecules of great interest, ${ }^{1}$ arguably owing to their increasing presence in pharmaceutical applications, ${ }^{2}$ molecular materials, ${ }^{3}$ use as synthetic precursors, ${ }^{4}$ and overall intriguing physical and chemical properties. In particular, the fulvene motif is a useful handle to probe aromaticity in what has been previously called an aromatic chameleon. ${ }^{5}$ While there are synthetic methods available for their preparation, most modern methods have become quite complex, using precursors and intermediates that are difficult to synthesize and/or expensive. Furthermore, many preparations use metal catalysts that must be fully removed before any applied use of the fulvene. ${ }^{6}$ Recently, there has been an effort to focus on cyclization reactions of enediynes, ${ }^{7}$ alkynyl derivatives, ${ }^{8}$ and transition-metal-based transformations ${ }^{9}$ to obtain these valuable functionalized fulvenes. While these methods have greatly improved our abilities to access a wealth of benzofulvene-based motifs, there is arguably room in this mix for a simple and effective synthetic solution. To that end we have been engaged in modifying and optimizing a traditional route to the benzofulvene core ${ }^{10}$ by utilizing substituted indanones to produce known and many novel functionalized benzofulvenes in three simple synthetic steps (Scheme 1).

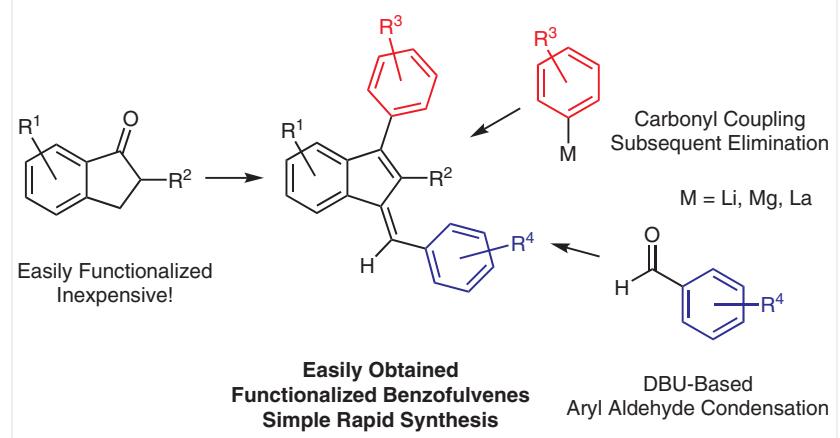

Scheme 1 Synthetic outline for the formation of functionalized benzofulvenes using simple, proven techniques

Our method utilizes reproducible, standard synthetic transformations that may have a more practical nature than many of the more complex synthetic steps currently being reported. Finally, our method is highly regioselective.

As illustrated in Scheme 1, our synthetic route begins with subjecting commercially available indanone to simple carbonyl coupling with various nucleophilic organometallic reagents (e.g., organolithium, organolanthanum, Grignard). We then subject the crude indanol mixture to known procedures utilizing catalytic iodine ${ }^{11}$ or diethylphosphitebased $^{12}$ elimination to produce the key indene intermediate. Our final synthetic step is a novel DBU mediated microwave coupling, to attach various benzaldehydes in good yield in a rapid and mild reaction (ca. 10 minutes $60^{\circ} \mathrm{C}$ ). Herein, we describe our work synthesizing a variety of functionalized benzofulvenes via a simple and mild method.

We began our synthetic process by optimizing the coupling of phenylindene with 4-methylbenzaldehyde (Table 1 ) by probing a variety of nucleophilic organometallic spe- 
cies to mediate the condensation. We found that yields were acceptable using an organolithium reagent in THF (entries 1 and 2). Upon switching to 2-MeTHF the yield was much improved relative to all other solvents investigated (entries 2-5). It is important to note that 2-MeTHF is considered a green solvent as it can be produced from agricultural waste. ${ }^{13}$ Other common nucleophilic metal species did improve the yield (entries 6 and 7), but arguably not enough to justify the added expense and complexity. Finally, we investigated using DBU (entry 8) in a microwaveheated transformation. While the yield described in entry 3 was the highest, we had a number of issues regarding reproducibility while using organolithium reagents for the transformation. Additionally the air-free nature of nucleophilic organometallic reagents can be problematic. For these reasons, we opted to use DBU under ambient conditions, because the yield was comparable and we found the reproducibility to be robust. Finally, the synthesis using DBU was much more straightforward from an ease of reaction standpoint, keeping with our desired strategy.

Table 1 Optimization of the Benzofulvene Condensation Step ${ }^{\mathrm{a}}$<smiles>CC1C=C(c2ccccc2)c2ccccc21</smiles><smiles>Cc1ccc(C=O)cc1</smiles><smiles></smiles><smiles></smiles>

$>95: 5 \mathrm{E}$ to $Z$ d.r.

\begin{tabular}{lllll}
\hline Entry & Solvent & Temp. $\left({ }^{\circ} \mathrm{C}\right)$ & $\mathrm{R}^{1}$ & Yield (\%) \\
\hline 1 & THF & -78 to r.t. & $\mathrm{Li}$ & 57 \\
2 & THF & 0 to r.t. & $\mathrm{Li}$ & 52 \\
3 & 2-MeTHF & 0 to r.t. & $\mathrm{Li}$ & 83 \\
4 & Et $2 \mathrm{O}$ & 0 to r.t. & $\mathrm{Li}$ & 40 \\
5 & toluene & 0 to r.t. & $\mathrm{Li}$ & 21 \\
6 & 2-MeTHF & 0 to r.t. & $\mathrm{La}$ & 74 \\
7 & 2-MeTHF & 0 to r.t. & $\mathrm{Eu}$ & 65 \\
8 & 2-MeTHF & $60{ }^{\circ} \mathrm{C}$ & $\mathrm{H}$ & $72^{\mathrm{c}}$
\end{tabular}

${ }^{a}$ All organometallic species were produced in situ. Specific synthetic information can be found in the Supporting Information.

${ }^{b}$ All yields are from ${ }^{1} \mathrm{H}$ NMR analysis using a hexadecane internal standard. c DBU (1 equiv.) was used.

We began to develop our synthetic library by functionalizing the exocyclic aromatic portion of the benzofulvene motif, as well as the indene core (Scheme 2). We have demonstrated that highly sterically restricted units can be incorporated in moderate yield (2b and $\mathbf{2 d}$ ). Highly electron-withdrawing trifluoromethyl groups $(\mathbf{2 e})$ could also be incorporated without issue. Unfortunately, we observed a decrease in yield for $\mathbf{2 h}$.

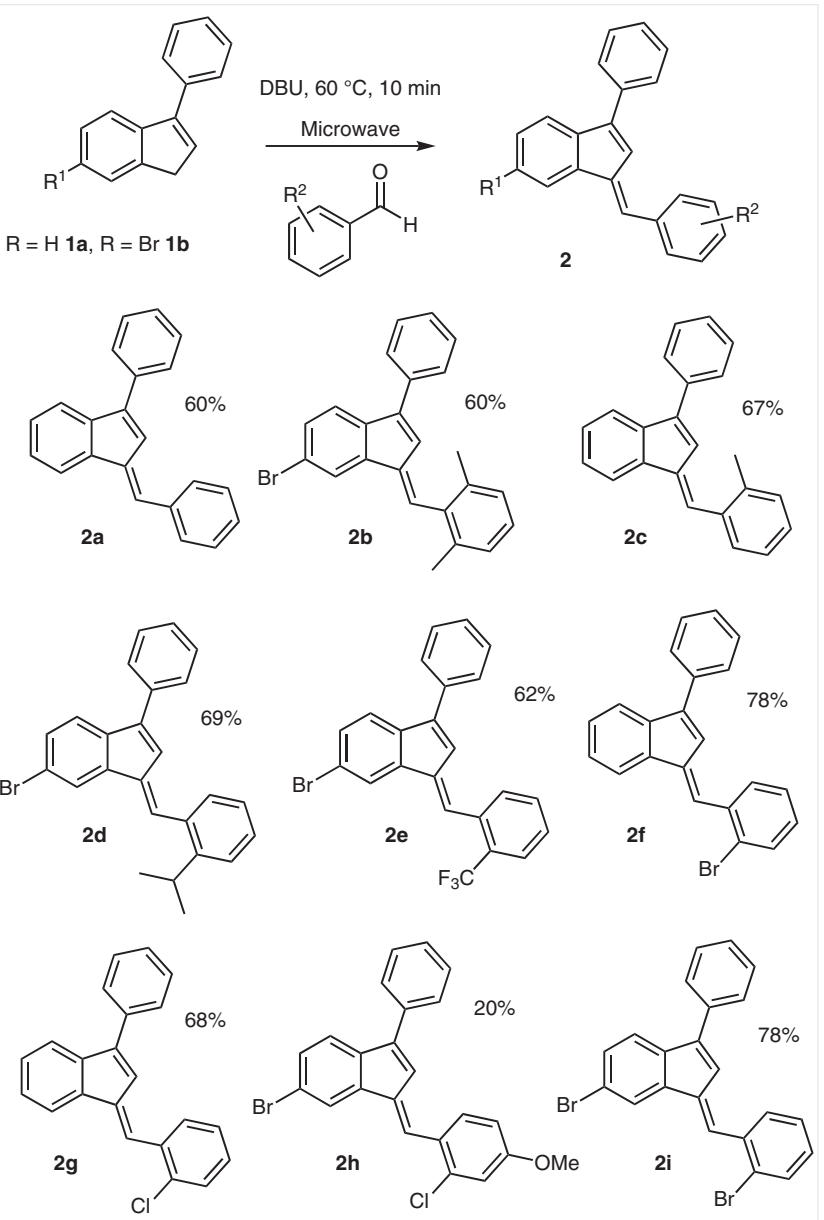

Scheme 2 Synthesis of functionalized benzofulvenes using our optimized procedure (see the Supporting Information for specifics regarding indene synthesis). All yields are for the isolated compound. Microwave heating was conducted using variable wattage.

We continued testing our method by incorporating electron-rich nucleophiles by transitioning to an organolanthanum addition during the indanone to indene synthesis (Scheme 3). When using standard carbonyl coupling reagents (i.e., organolithium or Grignard) we observed complete selectivity for the acid-base reaction. Upon switching to an organolanthanum coupling partner we were rewarded by improved yield for indene $\mathbf{1 d}$.

Once we successfully synthesized the methoxynaphthalene benzofulvene (Scheme 4, 3a) we aimed to synthesize a hydroxyl derivative using a $\mathrm{BBr}_{3}$ deprotection ${ }^{14}$ to produce 3b. We were delighted to witness this transformation without degradation of the fulvene motif. Furthermore, a hydroxyl derivative would allow for further reactivity through a variety of alkoxide-based transformations. Finally, we were able to produce fluoro derivative $\mathbf{3 c}$. 


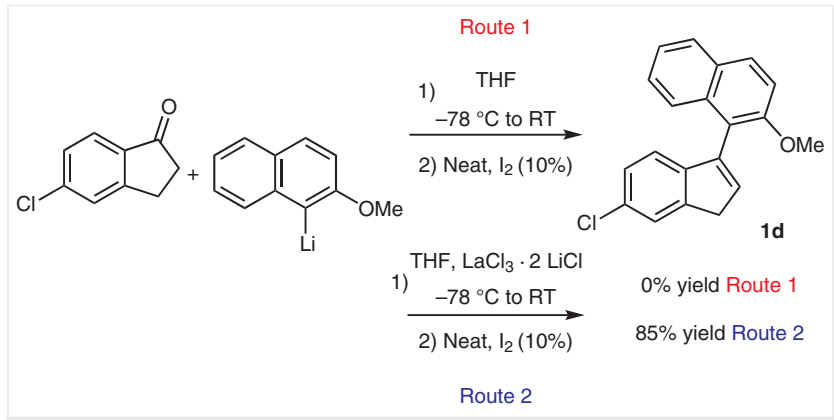

Scheme 3 Comparing organolanthanum and organolithium reactions for the creation of indene $\mathbf{1 d}$.

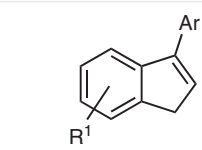

$\mathrm{R}^{1}=\mathrm{F} 1 \mathrm{c}, \mathrm{Cl}=\mathbf{1 d}$
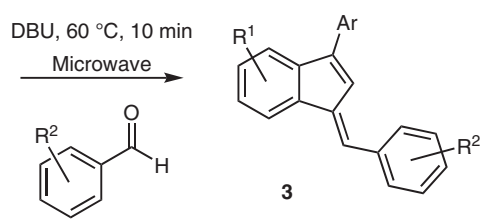

(c)

Scheme 4 Continued synthesis of highly functionalized benzofulvenes utilizing an organolanthanum coupling partner to indanone. The yield reported of $\mathbf{3} \mathbf{b}$ is after $\mathbf{a B B}{ }_{3}$ deprotection of $\mathbf{3 a}$. All yields are for the isolated compound. Microwave heating was conducted using variable wattage.

Additionally, we have provided preliminary evidence that our method is capable of producing larger benzofulvene dimers (Scheme 5). These dimers are a particularly interesting class of benzofulvenes because of their larger twodimensional conjugation. We were particularly pleased with the synthesis of more sterically restricted dimer $\mathbf{4 a}$, albeit in low yield.

Finally, in an effort to unambiguously determine structure, we obtained XRD structures for select compounds (Figure 1). Significantly, these will add to the limited number of fulvene-based structures available in the literature. Furthermore, our synthetic method allows for facile access to a wide variety of benzofulvenes that can be used to probe the interesting structural features related to the fulvene motif. Specifically, we wish to probe how dihedral angles around the exocyclic arene affect the bond length alternation of the fulvene. By producing a larger pool of crystal structures, we hope to probe how the bond length alternation of the fulvene is changed by the exocyclic arene. This

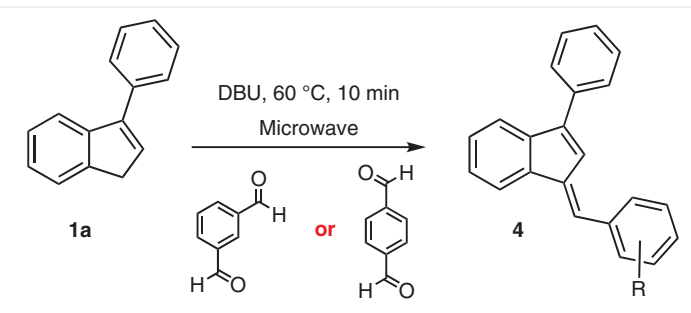<smiles>C1=C(c2ccccc2)c2ccccc2/C1=C/c1ccccc1/C=C/c1ccccc1-c1ccccc1</smiles><smiles>C1=C(c2ccccc2)c2ccccc2/C1=C/c1ccc(/C=C2\C=C(c3ccccc3)c3ccccc32)cc1</smiles>

Scheme $\mathbf{5}$ Synthesis of benzofulvene dimers $\mathbf{4 a}$ and $\mathbf{4 b}$. All yields are from the isolated compound. Microwave heating was conducted using variable wattage.

ongoing work may provide further empirical evidence to reveal the nature of the aromatic characteristics of the fulvene.

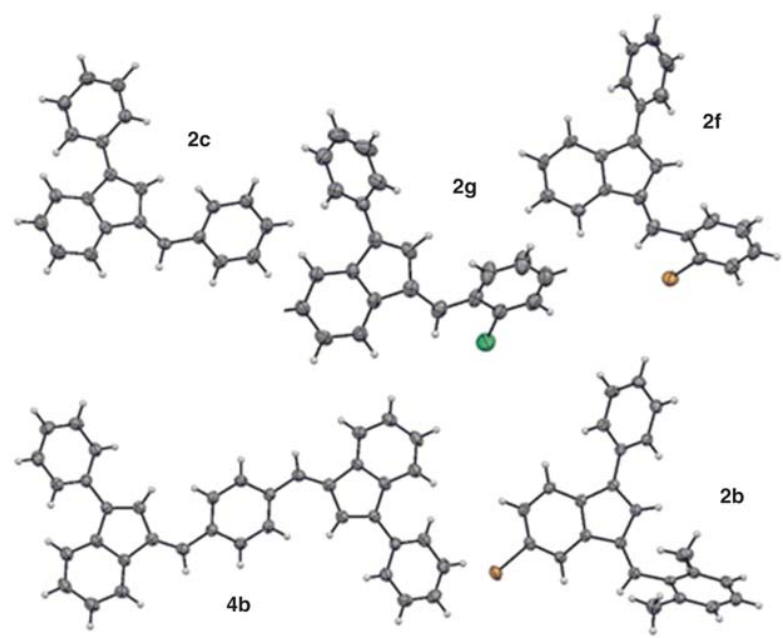

Figure 1 ORTEPs of representative benzofulvenes. CIFs and CCDC information can be obtained in the Supporting Information

In effect, we have established a simple and mild strategy for the synthesis of functionalized benzofulvenes. We have demonstrated broad functional group tolerance and the synthesis of some interesting benzofulvene motifs, including sterically bulky examples, an ability to incorporate EWGs and EDGs, and larger dimers using a simple and mild approach. 
Oxygen- and moisture-sensitive manipulations (indene synthesis) were performed by using standard Schlenk line techniques using anhydrous nitrogen gas.

Reagents 2-MeTHF, DBU, THF, various benzaldehydes, phenyl magnesium bromide and lanthanum(III) chloride were purchased from chemical manufacturers (i.e., Sigma Aldrich, TCI and Oakwood Chemical) and used without further purification.

${ }^{1} \mathrm{H}$ and ${ }^{13} \mathrm{C}$ NMR spectra were measured with a Bruker $500 \mathrm{MHz}$ Avance III NMR Spectrometer. All spectra were referenced to the respective solvent of $\mathrm{CDCl}_{3}$. GCMS was acquired with a HP GCMS with auto sampler. HRMS was obtained by the University of Michigan's Mass Spectrometry Facility. Microwave heating was performed with a Biotage Emrys Optimizer $300 \mathrm{~W}$ synthesizer. All microwave heating was performed in a sealed reaction vessel under ambient conditions using variable watt heating.

\section{Synthesis of Functionalized Benzofulvenes 2a-4b}

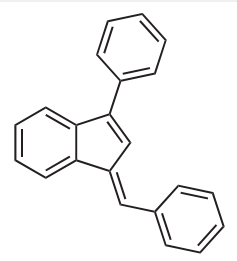

Figure 2

\section{Benzofulvene 2a (42788-18-1; Figure 2) $)^{15}$}

To a $10 \mathrm{~mL}$ microwave vial was added, indene $\mathbf{1 a}(0.135 \mathrm{~g}, 0.70$ mmol), benzaldehyde ( $0.075 \mathrm{~g}, 0.52 \mathrm{mmol}), 2-\mathrm{MeTHF}(1.0 \mathrm{~mL})$, and $\operatorname{DBU}(0.110 \mathrm{~g}, 0.70 \mathrm{mmol})$ and sealed. The mixture was stirred at $60{ }^{\circ} \mathrm{C}$ for 10 minutes by microwave heating. Once the reaction had finished, the crude mixture was flushed through a silica plug and the solvent removed under reduced pressure. The crude oil was subjected to flash chromatography (hexanes; $R_{f}=0.9$ ). The solvent was removed under reduced pressure to give a red oil.

Yield: $0.175 \mathrm{~g}(0.13 \mathrm{mmol}, 60 \%)$.

${ }^{1} \mathrm{H} \mathrm{NMR}\left(\mathrm{CDCl}_{3}, 500 \mathrm{MHz}\right): \delta=7.87-7.85(\mathrm{~m}, 1 \mathrm{H}), 7.79(\mathrm{~d}, J=8 \mathrm{~Hz}$, $2 \mathrm{H}), 7.74(\mathrm{~d}, J=8 \mathrm{~Hz}, 2 \mathrm{H}), 7.70-7.68(\mathrm{~m}, 1 \mathrm{H}), 7.61(\mathrm{~s}, 1 \mathrm{H}), 7.57-7.38$ $(\mathrm{m}, 8 \mathrm{H}), 7.24(\mathrm{~s}, 1 \mathrm{H})$

X-ray diffraction yielded crystal structure (CCDC 1835910).

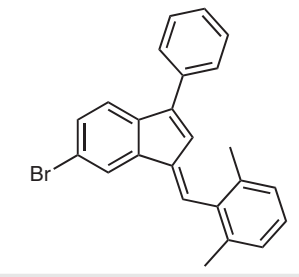

Figure 3

\section{Benzofulvene 2b (Figure 3)}

The procedure for $\mathbf{2 a}$ was followed using indene $\mathbf{1 b}(0.100 \mathrm{~g}, 0.37$ mmol), 2,6-dimethylbenzaldehyde (0.049 g, $0.37 \mathrm{mmol}), 2$-MeTHF $(1.0 \mathrm{~mL})$, and DBU $(0.056 \mathrm{~g}, 0.37 \mathrm{mmol})$. The crude oil was subjected to flash chromatography (hexane; $R_{f}=0.9$ ). The solvent was removed under reduced pressure to give a crystalline orange solid.

Yield: $0.084 \mathrm{~g}$ (0.22 mmol, 60\%); $\mathrm{mp} 154-160{ }^{\circ} \mathrm{C}$.
${ }^{1} \mathrm{H}$ NMR $\left(\mathrm{CDCl}_{3}, 500 \mathrm{MHz}\right): \delta=7.99(\mathrm{~s}, 1 \mathrm{H}), 7.63(\mathrm{~d}, J=8 \mathrm{~Hz}, 2 \mathrm{H})$, 7.53-7.42 (m, $6 \mathrm{H}), 7.24-7.17$ (m, $3 \mathrm{H}), 6.47$ (s, $1 \mathrm{H}), 2.35$ (s, $6 \mathrm{H})$

${ }^{13} \mathrm{C}$ NMR $\left(\mathrm{CDCl}_{3}, 125 \mathrm{MHz}\right): \delta=145.9,140.5,140.4,139.5,136.4$, $135.4,135.0,130.0,128.7,128.6,128.3,127.7,127.5$ ( 2 signals overlap), 124.4, 122.9, 121.5, 119.6, 20.9.

GC-MS EI: $m / z[\mathrm{M}]^{+}$calcd: 386.07 ; found: $386 ; m / z[\mathrm{M}+2]^{+}$calcd.: 388.06; found: 388 .

HRMS EI: $m / z$ [M] $]^{+}$calcd: 386.0670; found: 386.0663 .

X-ray diffraction yielded crystal structure (CCDC 1835914).

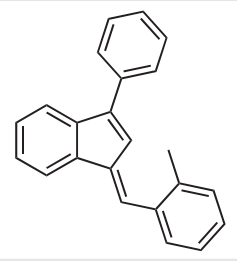

Figure 4

\section{Benzofulvene 2c (Figure 4)}

The procedure for $\mathbf{2 a}$ was followed using indene $\mathbf{1 a}(0.100 \mathrm{~g}, 0.52$ $\mathrm{mmol}), o$-tolualdehyde $(0.073 \mathrm{~g}, 0.52 \mathrm{mmol}), 2-\operatorname{MeTHF}(1.1 \mathrm{~mL})$, and $\operatorname{DBU}(0.081 \mathrm{~g}, 0.53 \mathrm{mmol})$. The crude oil was subjected to flash chromatography (95:5, hexane/EtOAc; $R_{f}=0.9$ ). The solvent was removed under reduced pressure to give a crystalline orange oil/solid.

Yield: $0.109 \mathrm{~g}(0.13 \mathrm{mmol}, 67 \%)$.

${ }^{1} \mathrm{H} \mathrm{NMR}\left(\mathrm{CDCl}_{3}, 500 \mathrm{MHz}\right): \delta=7.91-7.89(\mathrm{~m}, 1 \mathrm{H}), 7.78-7.76(\mathrm{~m}, 3 \mathrm{H})$, 7.71-7.67 (m, $2 \mathrm{H}), 7.55-7.52(\mathrm{~m}, 3 \mathrm{H}), 7.47-7.35(\mathrm{~m}, 5 \mathrm{H}), 7.07$ (s, $1 \mathrm{H}), 2.54(\mathrm{~s}, 3 \mathrm{H})$

${ }^{13} \mathrm{C}$ NMR $\left(\mathrm{CDCl}_{3}, 125 \mathrm{MHz}\right): \delta=147.0,141.2,139.8,138.4,137.5$, $136.1,135.7,131.1,130.2,128.6,128.3,128.0,127.7,127.4,127.0$, 126.0, 125.4, 123.8, 120.3, 119.4, 20.1.

GC-MS EI: $m / z$ [M] $]^{+}$calcd.: 294.14; found: 294 .

HRMS EI: $m / z$ [ M] $]^{+}$calcd.: 294.1409; found: 294.1402.

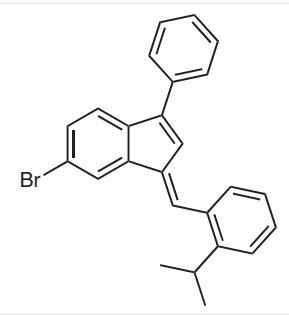

Figure 5

\section{Benzofulvene 2d (Figure 5)}

The procedure for $\mathbf{2 a}$ was followed using indene $\mathbf{1 b}(0.099 \mathrm{~g}, 0.37$ $\mathrm{mmol}), 2$-isopropylbenzaldehyde $(0.055 \mathrm{~g}, 0.37 \mathrm{mmol}), 2-\mathrm{MeTHF}(1.0$ $\mathrm{mL})$, and DBU $(0.056 \mathrm{~g}, 0.37 \mathrm{mmol})$. The crude oil was subjected to flash chromatography (hexane; $R_{f}=0.9$ ). The solvent was removed under reduced pressure to give an orange/red oil.

Yield: $0.102 \mathrm{~g}(0.25 \mathrm{mmol}, 69 \%)$.

${ }^{1} \mathrm{H}$ NMR $\left(\mathrm{CDCl}_{3}, 500 \mathrm{MHz}\right): \delta=7.92(\mathrm{~d}, J=1 \mathrm{~Hz}, 1 \mathrm{H}), 7.80(\mathrm{~s}, 1 \mathrm{H}), 7.64$ (d, $J=7 \mathrm{~Hz}, 2 \mathrm{H}), 7.52-7.37(\mathrm{~m}, 9 \mathrm{H}), 6.91(\mathrm{~d}, J=1 \mathrm{~Hz}, 1 \mathrm{H}$ ), 3.33 (sept, $J=7 \mathrm{~Hz}, 1 \mathrm{H}), 1.30(\mathrm{~d}, J=7 \mathrm{~Hz}, 6 \mathrm{H})$. 
${ }^{13} \mathrm{C}$ NMR $\left(\mathrm{CDCl}_{3}, 125 \mathrm{MHz}\right): \delta=148.1,146.2,140.3,139.9,139.2$, 135.2, 134.5, 131.6, 130.0, 129.0, 128.7, 128.5, 128.2, 127.5, 125.7, $125.2,124.1,122.8,121.5,119.4,30.2,23.5$.

GC-MS EI: $m / z$ [M] ${ }^{+}$calcd.: 400.08 ; found: $400 ; m / z[\mathrm{M}+2]^{+}$calcd.: 402.08; found: 402 .

HRMS EI: $m / z$ [M] $]^{+}$calcd.: 400.0827; found: 400.0828 .

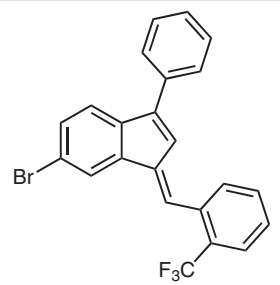

Figure 6

\section{Benzofulvene 2e (Figure 6)}

The procedure for $\mathbf{2 a}$ was followed using indene $\mathbf{1 b}(0.099 \mathrm{~g}, 0.37$ $\mathrm{mmol}), 2$-trifluoromethylbenzaldehyde $(0.064 \mathrm{~g}, 0.37 \mathrm{mmol}), 2$ MeTHF (1.0 mL), and DBU $(0.056 \mathrm{~g}, 0.37 \mathrm{mmol})$. The crude oil was subjected to flash chromatography (hexane; $R_{f}=0.9$ ). The solvent was removed under reduced pressure to give a crystalline orange solid.

Yield: $0.098 \mathrm{~g}$ (0.23 mmol, 62\%); mp 118-122 ${ }^{\circ} \mathrm{C}$.

${ }^{1} \mathrm{H}$ NMR $\left(\mathrm{CDCl}_{3}, 500 \mathrm{MHz}\right): \delta=7.92(\mathrm{~d}, J=1 \mathrm{~Hz}, 1 \mathrm{H}), 7.79-7.78(\mathrm{~m}$, $2 \mathrm{H}), 7.71-7.62(\mathrm{~m}, 4 \mathrm{H}), 7.51-7.40(\mathrm{~m}, 6 \mathrm{H}), 6.83(\mathrm{~s}, 1 \mathrm{H})$.

${ }^{13} \mathrm{C}$ NMR $\left(\mathrm{CDCl}_{3}, 125 \mathrm{MHz}\right): \delta=147.8,140.7,140.1,135.1,134.9$, 132.8, 131.7, 130.6, 129.1 (quartet, ${ }^{2} J=30 \mathrm{~Hz}$ ), 128.7, 128.6, 128.6 (quartet, ${ }^{3} \mathrm{~J}$ could not be determined due to signal overlap), 128.2, $127.5,126.1$ (quartet, ${ }^{3} \mathrm{~J}=5 \mathrm{~Hz}$ ), 124.9, 123.2, 123.1, 121.7, 119.9. ${ }^{1 \mathrm{~J}} \mathrm{R}-$ $\mathrm{CF}_{3}$ could not be determined due to signal overlap.

GCMS EI: $m / z$ [M] ${ }^{+}$calcd.: 426.02 ; found: $426 ; m / z[\mathrm{M}+2]^{+}$calcd.: 428.02; found: 428 .

HRMS EI: $m / z$ [M] $]^{+}$calcd.: 426.0231; found: 426.0228 .

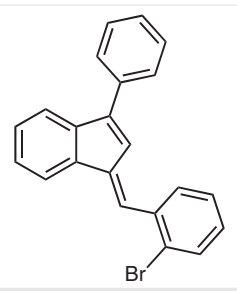

Figure 7

\section{Benzofulvene 2f (Figure 7)}

The procedure for $\mathbf{2 a}$ was followed using indene $\mathbf{1 a}(0.100 \mathrm{~g}, 0.52$ $\mathrm{mmol}$ ), 2-bromobenzaldehyde (0.096 g, $0.52 \mathrm{mmol}), 2$-MeTHF (1.0 $\mathrm{mL})$, and DBU $(0.082 \mathrm{~g}, 0.52 \mathrm{mmol})$. The crude oil was subjected to flash chromatography (hexane; $R_{f}=0.9$ ). The solvent was removed under reduced pressure to give a crystalline orange solid.

Yield: $0.15 \mathrm{~g}$ (0.40 mmol, $78 \%)$; $\mathrm{mp} 108-111{ }^{\circ} \mathrm{C}$.

${ }^{1} \mathrm{H} \mathrm{NMR}\left(\mathrm{CDCl}_{3}, 500 \mathrm{MHz}\right): \delta=7.73-7.71(\mathrm{~m}, 1 \mathrm{H}), 7.57-7.53(\mathrm{~m}, 5 \mathrm{H})$, 7.49-7.46 (m, $1 \mathrm{H}), 7.36-7.19(\mathrm{~m}, 6 \mathrm{H}), 7.10-7.06(\mathrm{~m}, 1 \mathrm{H}), 6.79(\mathrm{~s}$, $1 \mathrm{H})$.

${ }^{13} \mathrm{C}$ NMR $\left(\mathrm{CDCl}_{3}, 125 \mathrm{MHz}\right): \delta=148.0,141.1,140.6,138.2,137.0$, 135.5, 132.9, 132.4, 129.4, 128.7, 128.2, 127.8, 127.3, 127.1, 127.0, $125.7,125.1,122.9,120.4,119.8$
GC-MS EI: $m / z[\mathrm{M}]^{+}$calcd.: 358.04 ; found: $358 ; m / z[\mathrm{M}+2]^{+}$calcd.: 360.03; found: 360 .

HRMS EI: $m / z$ [M] calcd.: 358.0357; found: 358.0351 .

X-ray diffraction yielded crystal structure (CCDC 1835913).

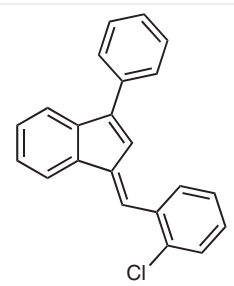

Figure 8

\section{Benzofulvene 2g (Figure 8)}

The procedure for $\mathbf{2 a}$ was followed using indene $\mathbf{1 a}(0.100 \mathrm{~g}, 0.52$ $\mathrm{mmol}), 2$-chlorobenzaldehyde $(0.076 \mathrm{~g}, 0.52 \mathrm{mmol}), 2$-MeTHF $(1.0$ $\mathrm{mL})$, and DBU $(0.082 \mathrm{~g}, 0.52 \mathrm{mmol})$. The crude oil was subjected to flash chromatography (hexane; $R_{f}=0.9$ ). The solvent was removed under reduced pressure to give a crystalline orange solid.

Yield: $0.110 \mathrm{~g}(0.35 \mathrm{mmol}, 68 \%) ; \mathrm{mp} 90-95{ }^{\circ} \mathrm{C}$.

${ }^{1} \mathrm{H} \mathrm{NMR}\left(\mathrm{CDCl}_{3}, 500 \mathrm{MHz}\right): \delta=7.90-7.88(\mathrm{~m}, 1 \mathrm{H}), 7.80(\mathrm{~s}, 1 \mathrm{H}), 7.76-$ $7.74(\mathrm{~m}, 3 \mathrm{H}), 7.67-7.65(\mathrm{~m}, 1 \mathrm{H}), 7.54-7.50(\mathrm{~m}, 3 \mathrm{H}), 7.47-7.44(\mathrm{~m}$, $1 \mathrm{H}), 7.41-7.32(\mathrm{~m}, 4 \mathrm{H}), 7.00(\mathrm{~s}, 1 \mathrm{H})$.

${ }^{13} \mathrm{C}$ NMR $\left(\mathrm{CDCl}_{3}, 125 \mathrm{MHz}\right): \delta=148.1,141.1,140.7,138.3,135.5$, 135.3, 134.8, 132.3, 129.7, 129.3, 128.6, 128.2, 127.8, 127.1, 126.7, $125.7,124.6,122.9,120.4,119.8$.

GC-MS EI: $m / z$ [M] $]^{+}$calcd.: 314.09 ; found: $314 ; m / z[\mathrm{M}+2]^{+}$calcd.: 316.08; found: 316 .

HRMS EI: $m / z$ [M] calcd.: 314.0862; found: 314.0860 .

$\mathrm{X}$-ray diffraction yielded crystal structure (CCDC 1835911).

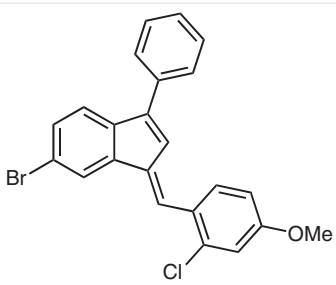

Figure 9

\section{Benzofulvene 2h (Figure 9)}

The procedure for $\mathbf{2 a}$ was followed using indene $\mathbf{1 b}(0.100 \mathrm{~g}, 0.37$ $\mathrm{mmol}), 2$-chloro-4-methoxybenzaldehyde (0.063 g, $0.37 \mathrm{mmol}), 2$ MeTHF $(1.0 \mathrm{~mL})$, and DBU $(0.056 \mathrm{~g}, 0.37 \mathrm{mmol})$. The crude oil was subjected to flash chromatography (hexane; $R_{f}=0.9$ ). The solvent was removed under reduced pressure to give a crystalline orange solid.

Yield: $0.031 \mathrm{~g}(0.074 \mathrm{mmol}, 20 \%) ; \mathrm{mp} 120-123^{\circ} \mathrm{C}$.

${ }^{1} \mathrm{H}$ NMR $\left(\mathrm{CDCl}_{3}, 500 \mathrm{MHz}\right): \delta=7.92(\mathrm{~s}, 1 \mathrm{H}), 7.68-7.63(\mathrm{~m}, 3 \mathrm{H}), 7.50-$ $7.38(\mathrm{~m}, 6 \mathrm{H}), 7.04(\mathrm{~d}, J=3 \mathrm{~Hz}, 1 \mathrm{H}), 6.96(\mathrm{~s}, 1 \mathrm{H}), 6.91-6.89(\mathrm{~m}, 1 \mathrm{H})$, $3.86(\mathrm{~s}, 3 \mathrm{H})$.

${ }^{13} \mathrm{C}$ NMR $\left(\mathrm{CDCl}_{3}, 125 \mathrm{MHz}\right): \delta=160.5,146.8,140.5,139.5,138.1$, 136.0, 135.2, 133.0, 130.0, 128.7, 128.4, 127.5, 127.1, 125.7, 123.1, 122.9, 121.6, 119.6, 115.1, 113.4, 55.7.

HRMS EI: $m / z$ [M]+ calcd: 422.0073; found: 422.0080 . 


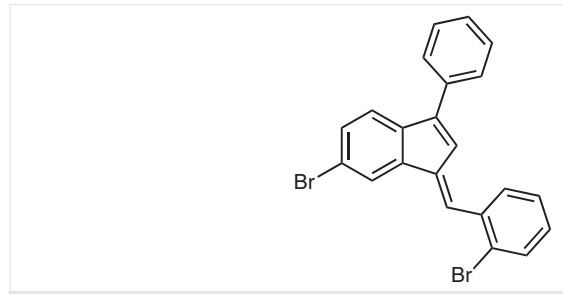

Figure 10

\section{Benzofulvene 2i (Figure 10)}

The procedure for $\mathbf{2 a}$ was followed using indene $\mathbf{1 b}(0.100 \mathrm{~g}, 0.37$ $\mathrm{mmol}), 2$-bromobenzaldehyde $(0.068 \mathrm{~g}, 0.37 \mathrm{mmol}), 2$-MeTHF (1.0 $\mathrm{mL})$, and DBU (0.056 g, $0.37 \mathrm{mmol})$. The crude oil was subjected to flash chromatography (98:2, hexane/EtOAc). The solvent was removed under reduced pressure to give a crystalline orange solid.

Yield: $0.13 \mathrm{~g}$ (0.29 mmol, 78\%); $\mathrm{mp} 114-119{ }^{\circ} \mathrm{C}$.

${ }^{1} \mathrm{H} \mathrm{NMR}\left(\mathrm{CDCl}_{3}, 500 \mathrm{MHz}\right): \delta=7.93(\mathrm{~s}, 1 \mathrm{H}), 7.68-7.62(\mathrm{~m}, 5 \mathrm{H}), 7.48-$ $7.44(\mathrm{~m}, 4 \mathrm{H}), 7.42-7.38(\mathrm{~m}, 3 \mathrm{H}), 6.90(\mathrm{~s}, 1 \mathrm{H})$.

${ }^{13} \mathrm{C} \mathrm{NMR}\left(\mathrm{CDCl}_{3}, 125 \mathrm{MHz}\right): \delta=147.4,140.2,139.9,139.6,136.6$, 135.0, 133.0, 132.4, 130.4, 129.8, 128.7, 128.5, 128.2, 127.5, 127.4, $125.2,123.2,123.1,121.6,119.8$.

GCMS EI: $m / z$ [M]+ calcd.: 435.95; found: 436 .

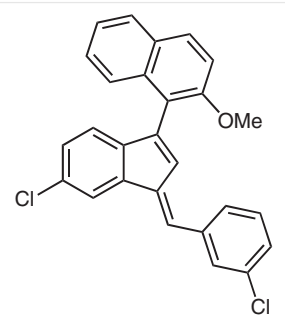

Figure 11

\section{Benzofulvene 3a (Figure 11)}

The procedure for $\mathbf{2 a}$ was followed using indene $\mathbf{1 d}(0.150 \mathrm{~g}, 0.49$ $\mathrm{mmol}), 3$-chlorobenzaldehyde $(0.0687 \mathrm{~g}, 0.49 \mathrm{mmol}), 2$-MeTHF (1.0 $\mathrm{mL})$, and DBU $(0.0744 \mathrm{~g}, 0.49 \mathrm{mmol})$. The crude oil was subjected to flash chromatography (95:5, hexane/EtOAc; $\left.R_{f}=0.9\right)$. The solvent was removed under reduced pressure to give a crystalline orange solid.

Yield: $0.147 \mathrm{~g}$ (70\%); $\mathrm{mp} 135-140{ }^{\circ} \mathrm{C}$.

${ }^{1} \mathrm{H} \mathrm{NMR}\left(\mathrm{CDCl}_{3}, 500 \mathrm{MHz}\right): \delta=7.95(\mathrm{~d}, J=9 \mathrm{~Hz}, 1 \mathrm{H}), 7.87-7.85(\mathrm{~m}$, $1 \mathrm{H}), 7.78-7.75$ (m, $2 \mathrm{H}), 7.65$ (s, $1 \mathrm{H}), 7.54-7.52$ (d, J = 9 Hz, $1 \mathrm{H}), 7.46$ (s, $1 \mathrm{H}), 7.42-7.31(\mathrm{~m}, 5 \mathrm{H}), 7.19-7.16(\mathrm{~m}, 1 \mathrm{H}), 7.12(\mathrm{~s}, 1 \mathrm{H}), 6.83(\mathrm{~d}, J$ $=8 \mathrm{~Hz}, 1 \mathrm{H}), 3.89(\mathrm{~s}, 3 \mathrm{H})$.

${ }^{13} \mathrm{C}$ NMR $\left(\mathrm{CDCl}_{3}, 125 \mathrm{MHz}\right): \delta=154.6,143.4,141.3,139.8,139.3$, $138.5,134.8,133.1,131.6,130.1,130.0,129.9,129.2,128.5,128.4$, 128.1, 127.5, 127.2, 126.7, 126.5, 125.2, 123.8, 121.7, 119.7, 113.5, 56.6. One aromatic signal overlaps.

HRMS EI: $m / z$ [M] calcd.: 428.0735; found: 428.0739 .

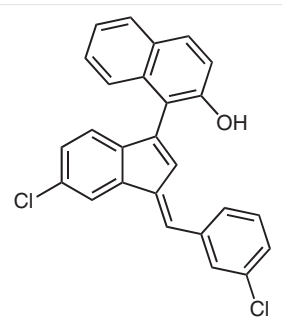

Figure 12

\section{Benzofulvene 3b (Figure 12)}

To a dried and flushed flask was added benzofulvene 3a $(125 \mathrm{mg}$, $0.292 \mathrm{mmol}$ ) and anhydrous dichloromethane $(3 \mathrm{~mL})$. The solution was cooled to $0{ }^{\circ} \mathrm{C}$, and boron tribromide $\left(1.0 \mathrm{M}\right.$ in $\left.\mathrm{CH}_{2} \mathrm{Cl}_{2}, 0.32 \mathrm{~mL}\right)$ was added dropwise. The reaction was warmed to r.t. and stirred overnight. Upon completion, the reaction was worked up with sodium thiosulfate ( $25 \mathrm{~mL}, 10 \%$ aqueous) and EtOAc $(20 \mathrm{~mL})$. The organic layer was dried $\left(\mathrm{MgSO}_{4}\right.$ anhydrous) and concentrated under reduced pressure. The crude oil was then purified by flash chromatography (50:50, hexane/EtOAc; $R_{f}=0.6$ ) to give a bright orange solid.

Yield: $76.4 \mathrm{mg}(63 \%) ; \mathrm{mp} 120-122{ }^{\circ} \mathrm{C}$.

${ }^{1} \mathrm{H}$ NMR $\left(\mathrm{CDCl}_{3}, 500 \mathrm{MHz}\right): \delta=7.86-7.83(\mathrm{~m}, 2 \mathrm{H}), 7.77(\mathrm{~s}, 1 \mathrm{H}), 7.62$ (s, $1 \mathrm{H}), 7.59-7.56(\mathrm{~m}, 1 \mathrm{H}), 7.54-7.50(\mathrm{~m}, 2 \mathrm{H}), 7.39-7.34(\mathrm{~m}, 4 \mathrm{H})$, $7.30(\mathrm{~d}, J=9 \mathrm{~Hz}, 1 \mathrm{H}), 7.23(\mathrm{~s}, 1 \mathrm{H}), 7.18(\mathrm{dd}, J=8,2 \mathrm{~Hz}, 1 \mathrm{H}), 6.86(\mathrm{~d}, J$ $=8 \mathrm{~Hz}, 1 \mathrm{H}), 5.60(\mathrm{~s}, \mathrm{br}, 1 \mathrm{H})$.

${ }^{13} \mathrm{C}$ NMR $\left(\mathrm{CDCl}_{3}, 125 \mathrm{MHz}\right): \delta=151.0,141.6,140.0,139.5,139.2$, 138.0, 134.9, 132.7, 132.6, 130.5, 130.2, 130.1, 129.0, 128.9, 128.4, $128.3,127.9,127.2,126.7,125.0,123.7,122.2,120.1,117.6,113.6$. One aromatic signal overlaps.

HRMS EI: $m / z$ [M] calcd.: 414.0578; found: 414.0550 .

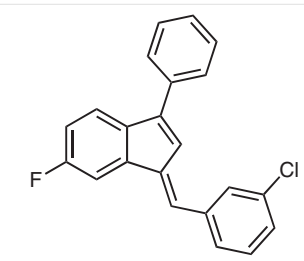

Figure 13

\section{Benzofulvene 3c (Figure 13)}

The procedure for $\mathbf{2 a}$ was followed using indene $\mathbf{1 c}(0.160 \mathrm{~g}, 1.1$ $\mathrm{mmol})$, 3-chlorobenzaldehyde (0.22 g, $1.0 \mathrm{mmol}), 2$-MeTHF (2.0 mL), and DBU $(0.170 \mathrm{~g}, 1.1 \mathrm{mmol})$. The crude oil was subjected to flash chromatography (95:10, hexane/EtOAc; $R_{f}=0.8$ ). The solvent was removed under reduced pressure to give a crimson solid.

Yield: $0.162 \mathrm{~g}$ (47\%); $\mathrm{mp} 110-114^{\circ} \mathrm{C}$.

${ }^{1} \mathrm{H} \mathrm{NMR}\left(\mathrm{CDCl}_{3}, 500 \mathrm{MHz}\right): \delta=7.68-7.66(\mathrm{~m}, 2 \mathrm{H}), 7.62(\mathrm{~s}, 1 \mathrm{H}), 7.51-$ $7.48(\mathrm{~m}, 4 \mathrm{H}), 7.44-7.41(\mathrm{~m}, 2 \mathrm{H}), 7.37-7.34(\mathrm{~m}, 3 \mathrm{H}), 7.04-7.00(\mathrm{~m}$, $2 \mathrm{H})$. 
${ }^{13} \mathrm{C}$ NMR $\left(\mathrm{CDCl}_{3}, 125 \mathrm{MHz}\right): \delta=163.1-161.2\left(\mathrm{~d}, J=244 \mathrm{~Hz}, \mathrm{C}-\mathrm{F}^{1} \mathrm{~J}\right)$, $147.8,140.9,140.8,139.5,138.4,136.7,135.2,134.7,129.9$ (d, $J=$ $\left.7 \mathrm{~Hz}, \mathrm{C}-\mathrm{F}{ }^{3} \mathrm{~J}\right), 128.7,128.5$ (d, $\left.J=7 \mathrm{~Hz}, \mathrm{C}-\mathrm{F}{ }^{3} \mathrm{~J}\right), 128.3,127.5,126.9$, $122.3\left(\mathrm{~d}, J=3 \mathrm{~Hz}, \mathrm{C}-\mathrm{F}^{4} J\right), 121.3,121.2,114.1-114.0$ (d, $J=23 \mathrm{~Hz}, \mathrm{C}-\mathrm{F}$ $\left.{ }^{2} \mathrm{~J}\right), 107.4-107.2\left(\mathrm{~d}, J=23 \mathrm{~Hz}, \mathrm{C}-\mathrm{F}^{2} \mathrm{~J}\right)$.

GC-MS EI: $m / z$ [M] ${ }^{+}$calcd.: 332.8; found: 332 .

HRMS EI: $m / z$ [M] $]^{+}$calcd.: 332.0768; found: 332.0767 .

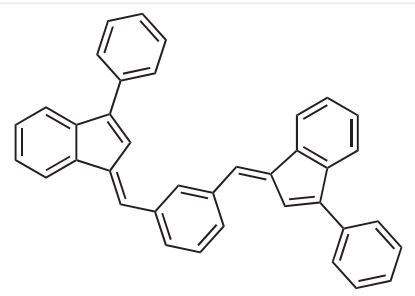

Figure 14

\section{Benzofulvene 4a (Figure 14)}

The procedure for $\mathbf{2 a}$ was followed using indene $\mathbf{1 a}(0.200 \mathrm{~g}, 1.0$ mmol), m-phthalaldehyde (0.070 g, $0.5 \mathrm{mmol}), 2$-MeTHF $(1.0 \mathrm{~mL})$, and DBU $(0.182 \mathrm{~g}, 1.2 \mathrm{mmol})$. The crude oil was subjected to flash chromatography $\left(95: 5\right.$, hexane/EtOAc; $\left.R_{f}=0.8\right)$. The solvent was removed under reduced pressure to give a yellow oil.

Yield: $0.040 \mathrm{~g}$ (16\%).

${ }^{1} \mathrm{H} \mathrm{NMR}\left(\mathrm{CDCl}_{3}, 500 \mathrm{MHz}\right): \delta=7.94(\mathrm{~s}, 1 \mathrm{H}), 7.83-7.81(\mathrm{~m}, 2 \mathrm{H}), 7.72-$ $7.71(\mathrm{~m}, 4 \mathrm{H}), 7.67-7.62(\mathrm{~m}, 4 \mathrm{H}), 7.57(\mathrm{~s}, 2 \mathrm{H}), 7.54-7.53(\mathrm{~m}, 1 \mathrm{H})$, 7.48-7.44 (m, $4 \mathrm{H}), 7.42-7.40(\mathrm{~m}, 2 \mathrm{H}), 7.37-7.34(\mathrm{~m}, 4 \mathrm{H}), 7.2(\mathrm{~s}$, $2 \mathrm{H})$.

${ }^{13} \mathrm{C}$ NMR $\left(\mathrm{CDCl}_{3}, 125 \mathrm{MHz}\right): \delta=148.0,140.8,139.8,138.8,137.6$, 135.6, 131.6, 130.0, 129.1, 128.6 (2 peaks overlapped), 128.2, 127.6, $127.5,127.4,125.6,122.8,120.4,119.5$.

HRMS EI: $m / z$ [M] calcd.: 482.2034; found: 482.2034 .

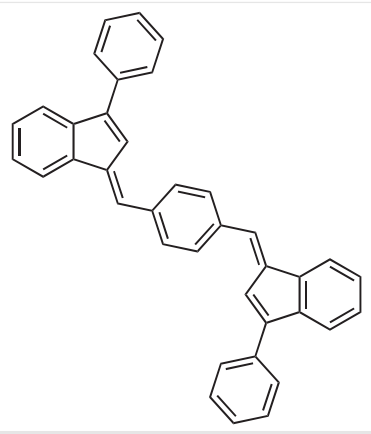

Figure 15

\section{Benzofulvene 4b (Figure 15)}

The procedure for $\mathbf{2 a}$ was followed using indene $\mathbf{1 a}(0.200 \mathrm{~g}, 1.0$ $\mathrm{mmol}), p$-phthalaldehyde $(0.070 \mathrm{~g}, 0.5 \mathrm{mmol}), 2-\mathrm{MeTHF}(1.0 \mathrm{~mL})$, and DBU $(0.185 \mathrm{~g}, 1.2 \mathrm{mmol})$. The crude oil was subjected to flash chromatography (95:5, hexane/EtOAc; $R_{f}=0.8$ ). The solvent was removed under reduced pressure to give a red solid.

Yield: $0.100 \mathrm{~g}$ (40\%); $\mathrm{mp} 212-214^{\circ} \mathrm{C}$.

${ }^{1} \mathrm{H} \mathrm{NMR}\left(\mathrm{CDCl}_{3}, 500 \mathrm{MHz}\right): \delta=7.82-7.80(\mathrm{~m}, 2 \mathrm{H}), 7.76$ (s, $\left.4 \mathrm{H}\right), 7.74-$ 7.73 (m, 4 H), 7.62-7.60 (2 H), 7.54 (s, $2 \mathrm{H}), 7.51-7.48$ (m, $5 \mathrm{H}), 7.43-$ $7.40(\mathrm{~m}, 3 \mathrm{H}), 7.34-7.32(\mathrm{~m}, 4 \mathrm{H}), 7.20(\mathrm{~s}, 2 \mathrm{H})$.
Full characterization data can be obtained from a prior publication. ${ }^{16}$

\section{Funding Information}

We would like to thank the PLU Division of Natural Sciences for funding this work.

\section{Acknowledgment}

We wish to thank the following individuals for their help with synthetic work: John Rubenkonig, Austin Erler, Michael Cargill, Valerie Lesniak, Gregg Lowery, and John Fordice.

\section{Supporting Information}

Supporting information for this article is available online at https://doi.org/10.1055/s-0037-1610366.

\section{References}

(1) Preethalayam, P.; Krishnan, K. S.; Thulasi, S.; Chand, S. S.; Joseph, J.; Nair, V.; Jaroschik, F.; Radhakrishnan, K. V. Chem. Rev. 2017, 117, 3930.

(2) (a) Licciardi, M.; Scialabba, C.; Giammona, G. J. Nanopart. Res. 2017, 19, 197. (b) Cappelli, A.; Paolino, M.; Grisci, G.; Razzano, V.; Giuliani, G.; Donati, A.; Bonechi, C.; Mendichi, R.; Battiato, S.; Samperi, F.; Scialabba, C.; Giammona, G.; Makovece, F.; Licciardi, M. Polym. Chem. 2016, 7, 6529.

(3) (a) Corrente, G. A.; Fabiano, E.; De Marco, L.; Accorsi, G.; Giannuzzi, R.; Cardone, A.; Gigli, G.; Ciccarella, G.; Capodilupo, A.-L. J. Mater. Sci.: Mater. Electron. 2017, 28, 8694. (b) Tortorella, S.; Talamo, M. M.; Cardone, A.; Pastore, M.; Angelis, F. J. Phys.: Condens. Matter 2016, 28, 074005. (c) Biani, F. F.; Reale, A.; Razzano, V.; Paolino, M.; Giuliani, G.; Donati, A.; Giorgi, G.; Mroz, W.; Piovanni, D.; Botta, C.; Cappelli, A. RSC Adv. 2018, 8, 10836.

(4) Donslund, B. S.; Nielsen, R. P.; Mønsted, S. M. N.; Jørgensen, K. A. Angew. Chem. 2016, 128, 11290.

(5) (a) Möllerstedt, H.; Carmen, M.; Crespo, R.; Ottosson, H. J. Am. Chem. Soc. 2004, 126, 13938. (b) Ayub, R.; El Bakouri, O.; Jorner, K.; Solà, M.; Ottosson, H. J. Org. Chem. 2017, 82, 6327.

(6) Bahramian, B.; Ma, Y.; Rohanizadeh, R.; Chrzanowski, W.; Dehghani, F. Green Chem. 2016, 18, 3740.

(7) Chen, S.; Li, L.; Sun, S.; Ding, Y.; Hu, A. Macromolecules 2017, 50, 534.

(8) García-García, P.; Sanjuán, A. M.; Rashid, M. A.; MartínezCuezva, A.; Fernández-Rodríguez, M. A.; Rodríguez, F.; Sanz, R. J. Org. Chem. 2017, 82, 1155.

(9) Shin, S.; Son, J.-U.; Choi, C.; Kim, S.; Lee, P. H. J. Org. Chem. 2016, $81,11706$.

(10) Böhme, T. M.; Keim, C.; Dannhardt, G.; Mutschler, E.; Lambrecht, G. Bioorg. Med. Chem. Lett. 2001, 11, 1241.

(11) Stavber, G.; Zupan, M.; Stavber, S. Tetrahedron Lett. 2006, 8463.

(12) Wang, T.; Hu, Y.; Zhang, S. Org. Biomol. Chem. 2010, 8, 2312.

(13) Aycock, D. F. Org. Process Res. Dev. 2007, 11, 156.

(14) Kosik, T. M.; Conrad, H. A.; Korich, A. L.; Lord, R. L. Eur. J. Org. Chem. 2015, 7460.

(15) Marcuzzi, F.; Melloni, G. Chim. Ind. (Rome, Italy) 1976, 58, 734s.

(16) Glass, A. C.; Cargill, M. ChemSpider Synthetic Pages 2015, SP 778. 